\title{
HODGE GROUPS OF CERTAIN SUPERELLIPTIC JACOBIANS
}

\author{
JiAngWei Xue AND Yuri G. Zarhin
}

Throughout this paper $K$ is a field of characteristic zero, $\bar{K}$ its algebraic closure and $\operatorname{Gal}(K)=\operatorname{Aut}(\bar{K} / K)$ the absolute Galois group of $K$. If $X$ is an abelian variety over $\bar{K}$ then we write $\operatorname{End}(X)$ for the ring of all its $\bar{K}$-endomorphisms and $\operatorname{End}^{0}(X)$ for the corresponding $\mathbf{Q}$-algebra $\operatorname{End}(X) \otimes \mathbf{Q}$; the notation $1_{X}$ stands for the identity automorphism of $X$.

Let $f(x) \in K[x]$ be a polynomial of degree $n \geq 3$ with coefficients in $K$ and without multiple roots, $\mathfrak{R}_{f} \subset \bar{K}$ the (n-element) set of roots of $f$ and $K\left(\Re_{f}\right) \subset \bar{K}$ the splitting field of $f$. We write $\operatorname{Gal}(f)=\operatorname{Gal}(f / K)$ for the Galois group $\operatorname{Gal}\left(K\left(\Re_{f}\right) / K\right)$ of $f$; it permutes roots of $f$ and may be viewed as a certain permutation group of $\mathfrak{R}_{f}$, i.e., as as a subgroup of the group $\operatorname{Perm}\left(\mathfrak{R}_{f}\right) \cong \mathbf{S}_{n}$ of permutation of $\mathfrak{R}_{f}$. $(\operatorname{Gal}(f)$ is transitive if and only if $f$ is irreducible.)

Suppose that $p$ is a prime that does not divide $n$ and a positive integer $q=p^{r}$ is a power of $p$. We write $C_{f, q}$ for the superelliptic $K$-curve $y^{q}=f(x)$ and $J\left(C_{f, q}\right)$ for its jacobian. Clearly, $J\left(C_{f, q}\right)$ is an abelian variety that is defined over $K$ and

$$
\operatorname{dim}\left(J\left(C_{f, q}\right)\right)=\frac{(n-1)(q-1)}{2} .
$$

Assume that $K$ contains a primitive $q$ th root of unity $\zeta_{q}$. In a series of papers $[12,14,15,16]$, one of the authors (Y.Z.) discussed the structure of $\operatorname{End}^{0}\left(J\left(C_{f, q}\right)\right)$, assuming that $n \geq 5$ and the Galois group $\operatorname{Gal}(f)$ of $f(x)$ over $K$ is, at least, doubly transitive. In particular, he proved that if $n \geq 5$ and $\operatorname{Gal}(f)$ coincides either with full symmetric group $\mathbf{S}_{n}$ or with alternating group $\mathbf{A}_{n}$ then $\operatorname{End}^{0}\left(J\left(C_{f, q}\right)\right)$ is (canonically) isomorphic to a product $\prod_{i=1}^{r} \mathbf{Q}\left(\zeta_{p^{i}}\right)$ of cyclotomic fields. (If $q=p$ then we proved that $\operatorname{End}\left(J\left(C_{f, p}\right)\right)=\mathbf{Z}\left[\zeta_{p}\right]$.) More precisely, if $q \neq p$ then the map $(x, y) \rightarrow\left(x, y^{p}\right)$ defines the map of curves $C_{f, q} \rightarrow C_{f, q / p}$, which induces (by Albanese functoriality) the surjective homomorphism $J\left(C_{f, q}\right) \rightarrow J\left(C_{f, q / p}\right)$ of abelian varieties over $K$; we write $J^{(f, q)}$ for the identity component of its kernel. (If $q=p$ then we put $J^{(f, q)}=$ $J\left(C_{f, p}\right)$.) One may check [16] that $J\left(C_{f, q}\right)$ is $K$-isogenous to the product $\prod_{i=1}^{r} J^{\left(f, p^{i}\right)}$ and the automorphism $\delta_{q}:(x, y) \mapsto\left(x, \zeta_{q} y\right)$ of $C_{f, q}$ gives rise to an embedding $\mathbf{Z}\left[\zeta_{q}\right] \hookrightarrow \operatorname{End}\left(J^{(f, q)}\right), \zeta_{q} \mapsto \delta_{q}$. What was actually proved in $[16,18]$ is that

$$
\mathbf{Z}\left[\zeta_{q}\right] \cong \mathbf{Z}\left[\delta_{q}\right]=\operatorname{End}\left(J^{(f, q)}\right)
$$

if $\operatorname{Gal}(f)=\mathbf{S}_{n}, p \geq 3$ and $n \geq 4$ or $\operatorname{Gal}(f)=\mathbf{A}_{n}$ and $n \geq 5$.

Let us assume that $K \subset \mathbf{C}$ and let the field $\bar{K}$ be the algebraic closure of $K$ in $\mathbf{C}$. This allows us to consider $J\left(C_{f, q}\right)$ and $J^{(f, q)}$ as complex abelian varieties. Our goal is to study the (reductive Q-algebraic connected) Hodge group $\operatorname{Hdg}=\operatorname{Hdg}\left(J^{(f, q)}\right)$ of $J^{(f, q)}$. Notice that when $q=2$ (i.e., in the hyperelliptic case) this group was

Received by the editors October 14, 2009. 
completely determined in [13] (when $f(x)$ has "large" Galois group). When $q>2$ we determined in our previous paper [9] the center of $\operatorname{Hdg}\left(J^{(f, q)}\right)$, also assuming that the Galois group of $f(x)$ is "large".

Let us assume that $q>2$. In order to describe our results let us recall that the jacobian $J\left(C_{f, q}\right)$ carries the canonical principal polarization that is invariant under all automorphisms (induced by automorphisms) of $C_{f, q}$. This implies that the induced polarization on the abelian subvariety $J^{(f, q)}$ is $\delta_{q}$-invariant. This polarization gives rise to the $\delta_{q}$-invariant nondegenerate alternating $\mathbf{Q}$-bilinear form

$$
\psi_{q}: \mathrm{H}_{1}\left(J^{(f, q)}, \mathbf{Q}\right) \times \mathrm{H}_{1}\left(J^{(f, q)}, \mathbf{Q}\right) \rightarrow \mathbf{Q}
$$

on the first rational homology group of the complex abelian variety $J^{(f, q)}$. On the other hand, $\mathrm{H}_{1}\left(J^{(f, q)}, \mathbf{Q}\right)$ carries the natural structure of $\mathbf{Q}\left[\delta_{q}\right] \cong \mathbf{Q}\left(\zeta_{q}\right)$-vector space. The $\delta_{q}$-invariance of $\psi_{q}$ implies that

$$
\psi_{q}(e x, y)=\psi_{q}(x, \bar{e} y) \quad \forall e \in \mathbf{Q}\left(\zeta_{q}\right) ; x, y \in \mathrm{H}_{1}\left(J^{(f, q)}, \mathbf{Q}\right) .
$$

Here $e \mapsto \bar{e}$ stands for the complex conjugation map. Let

$$
\mathbf{Q}\left(\zeta_{q}\right)^{+}=\left\{e \in \mathbf{Q}\left(\zeta_{q}\right) \mid \bar{e}=e\right\}
$$

be the maximal totally real subfield of the cyclotomic CM field $\mathbf{Q}\left(\zeta_{q}\right)$ and let

$$
\mathbf{Q}\left(\zeta_{q}\right)_{-}=\left\{e \in \mathbf{Q}\left(\zeta_{q}\right) \mid \bar{e}=-e\right\} .
$$

Pick a non-zero element $\alpha \in \mathbf{Q}\left(\zeta_{q}\right)_{-}$. Now the standard construction (see, for instance, [5, p. 531]) allows us to define the non-degenerate $\mathbf{Q}\left(\zeta_{q}\right)$-sesquilinear Hermitian form

such that

$$
\phi_{q}: \mathrm{H}_{1}\left(J^{(f, q)}, \mathbf{Q}\right) \times \mathrm{H}_{1}\left(J^{(f, q)}, \mathbf{Q}\right) \rightarrow \mathbf{Q}\left(\zeta_{q}\right)
$$

$$
\psi_{q}(x, y)=\operatorname{Tr}_{\mathbf{Q}\left(\zeta_{q}\right) / \mathbf{Q}}\left(\alpha \phi_{q}(x, y)\right) \forall x, y \in \mathrm{H}_{1}\left(J^{(f, q)}, \mathbf{Q}\right) .
$$

We write $\mathrm{U}\left(\mathrm{H}_{1}\left(J^{(f, q)}, \mathbf{Q}\right), \phi_{q}\right)$ for the unitary group of $\phi_{q}$ of the $\mathbf{Q}\left(\zeta_{q}\right)$-vector space $\mathrm{H}_{1}\left(J^{(f, q)}, \mathbf{Q}\right)$, viewed as an algebraic Q-subgroup of $\mathrm{GL}\left(\mathrm{H}_{1}\left(J^{(f, q)}, \mathbf{Q}\right)\right.$ ) (via Weil's restriction of scalars from $\mathbf{Q}\left(\zeta_{q}\right)^{+}$to $\mathbf{Q}$ (ibid). Since the Hodge group respects the polarization and commutes with endomorphisms of $J^{(f, q)}$,

$$
\operatorname{Hdg}\left(J^{(f, q)}\right) \subset \mathrm{U}\left(\mathrm{H}_{1}\left(J^{(f, q)}, \mathbf{Q}\right), \phi_{q}\right) .
$$

Our main result is the following statement.

Theorem 0.1. Suppose that $n \geq 4$ and $p$ is a prime that does not divide $n$. Let $f(x) \in \mathbf{C}[x]$ be a degree $n$ polynomial without multiple roots. Let $r$ be a positive integer and $q=p^{r}$. Suppose that there exists a subfield $K$ of $\mathbf{C}$ that contains all the coefficients of $f(x)$. Let us assume that $f(x)$ is irreducible over $K$ and the Galois group $\operatorname{Gal}(f)$ of $f(x)$ over $K$ is either $\mathbf{S}_{n}$ or $\mathbf{A}_{n}$. Assume additionally that either $n \geq 5$ or $n=4$ and $\operatorname{Gal}(f)=\mathbf{S}_{4}$.

Suppose that $n>q$ and one of the following three conditions holds:

(A) $q<n<2 q$;

(B) $p$ is odd and $n \not \equiv 1 \bmod q$;

(C) $p=2, n \not \equiv 1 \bmod q$ and $n \not \equiv q-1 \bmod 2 q$.

Then $\operatorname{Hdg}\left(J^{(f, q)}\right)=\mathrm{U}\left(\mathrm{H}_{1}\left(J^{(f, q)}, \mathbf{Q}\right), \phi_{q}\right)$.

Remark 0.2. The case of $q=p=3$ was earlier treated in [14]. 
Lefschetz's theorem about algebraicity of 2-dimensional Hodge classes and classical invariant theory for the unitary groups $[5$, Theorem 0 on p. 524 ; see also pp. $531-532$ ] imply the following corollary to Theorem 0.1 .

Corollary 0.3. Let $(n, p, q, f(x))$ satisfy the conditions of Theorem 0.1. Then every Hodge class on each self-product $\left(J^{(f, q)}\right)^{m}$ of $J^{(f, q)}$ can be presented as a linear combination with rational coefficients of products of divisor classes. In particular, the Hodge conjecture holds true for $\left(J^{(f, q)}\right)^{m}$.

The paper is organized as follows. In Section 1 we discuss Lie algebras of Hodge groups of complex abelian varieties. Its main result, Theorem 1.1 (that may be of independent interest) asserts that under certain conditions the semisimple part of the Hodge group (and its Lie algebra) is "as large as possible". We deduce Theorem 0.1 from Theorem 1.1, using auxiliary results from Section 2 concerning divisibility properties of certain arithmetic functions. In Section 3 we discuss linear reductive Lie algebras. The last Section contains the proof of Theorem 1.1.

\section{Complex abelian varieties}

Let $Z$ be a complex abelian variety of positive dimension and let $\Omega^{1}(Z)$ be the $\operatorname{dim}(Z)$-dimensional complex vector space of regular differential 1 -forms on $Z$. We write $\mathfrak{C}_{Z}$ for the center of the semisimple finite-dimensional Q-algebra $\operatorname{End}^{0}(Z)$. We write $\mathrm{H}_{1}(Z, \mathbf{Q})$ for its first rational homology group. It is well known that $\mathrm{H}_{1}(Z, \mathbf{Q})$ is a $2 \operatorname{dim}(Z)$-dimensional $\mathbf{Q}$-vector space.

The Q-algebra $\operatorname{End}^{0}(Z)$ acts faithfully on $\mathrm{H}_{1}(Z, \mathbf{Q})$. In particular, if $E$ is a subfield of $\operatorname{End}^{0}(Z)$ that contains the identity map then $\mathrm{H}_{1}(Z, \mathbf{Q})$ carries the natural structure of $E$-vector space of dimension

$$
d(Z, E)=\frac{2 \operatorname{dim}(Z)}{[E: \mathbf{Q}]}
$$

Let $\Sigma_{E}$ be the set of all field embeddings $\sigma: E \hookrightarrow \mathbf{C}$. It is well-known that

$$
\mathbf{C}_{\sigma}:=E \otimes_{E, \sigma} \mathbf{C}=\mathbf{C}, \quad E_{\mathbf{C}}=E \otimes_{\mathbf{Q}} \mathbf{C}=\prod_{\sigma \in \Sigma_{E}} E \otimes_{E, \sigma} \mathbf{C}=\prod_{\sigma \in \Sigma_{E}} \mathbf{C}_{\sigma} .
$$

If $\sigma \in \Sigma_{E}$ then we write $\bar{\sigma}$ for the complex-conjugate of $\sigma$. We write $X_{E}$ for the $\mathbf{Q}$-vector space of functions $\phi: \Sigma_{E} \rightarrow \mathbf{Q}$ with

$$
\phi(\bar{\sigma})+\phi(\sigma)=0 \forall \sigma \in \Sigma_{E} .
$$

If $E / \mathbf{Q}$ is Galois then $X_{E}$ carries the natural structure of $\operatorname{Gal}(E / \mathbf{Q})$-module.

Let $\operatorname{Lie}(Z)$ be the tangent space to the origin of $Z$; it is a $\operatorname{dim}(Z)$-dimensional $\mathbf{C}$ vector space. By functoriality, $\operatorname{End}^{0}(Z)$ and therefore $E$ act on $\operatorname{Lie}(Z)$ and therefore provide $\operatorname{Lie}(Z)$ with a natural structure of $E \otimes_{\mathbf{Q}} \mathbf{C}$-module. Clearly,

$$
\operatorname{Lie}(Z)=\bigoplus_{\sigma \in \Sigma_{E}} \mathbf{C}_{\sigma} \operatorname{Lie}(Z)=\oplus_{\sigma \in \Sigma_{E}} \operatorname{Lie}(Z)_{\sigma}
$$

where $\operatorname{Lie}(Z)_{\sigma}:=\mathbf{C}_{\sigma} \operatorname{Lie}(Z)=\{x \in \operatorname{Lie}(Z) \mid e x=\sigma(e) x \quad \forall e \in E\}$. Let us put $n_{\sigma}=n_{\sigma}(Z, E)=\operatorname{dim}_{\mathbf{C}_{\sigma}} \operatorname{Lie}(Z)_{\sigma}=\operatorname{dim}_{\mathbf{C}} \operatorname{Lie}(Z)_{\sigma}$. It is well-known that the natural $\operatorname{map} \Omega^{1}(Z) \rightarrow \operatorname{Hom}_{\mathbf{C}}(\operatorname{Lie}(Z), \mathbf{C})$ is an isomorphism. This allows us to define via duality the natural homomorphism $E \rightarrow \operatorname{End}_{\mathbf{C}}\left(\operatorname{Hom}_{\mathbf{C}}(\operatorname{Lie}(Z), \mathbf{C})\right)=\operatorname{End}_{\mathbf{C}}\left(\Omega^{1}(Z)\right)$. 
This provides $\Omega^{1}(Z)$ with a natural structure of $E \otimes_{\mathbf{Q}} \mathbf{C}$-module in such a way that $\Omega^{1}(Z)_{\sigma}:=\mathbf{C}_{\sigma} \Omega^{1}(Z) \cong \operatorname{Hom}_{\mathbf{C}}\left(\operatorname{Lie}(Z)_{\sigma}, \mathbf{C}\right)$. In particular,

$$
n_{\sigma}=\operatorname{dim}_{\mathbf{C}}\left(\operatorname{Lie}(Z)_{\sigma}\right)=\operatorname{dim}_{\mathbf{C}}\left(\Omega^{1}(Z)_{\sigma}\right)
$$

Let us consider the first complex homology group of $Z$

$$
\mathrm{H}_{1}(Z, \mathbf{C})=\mathrm{H}_{1}(Z, \mathbf{Q}) \otimes_{\mathbf{Q}} \mathbf{C},
$$

which is a $2 \operatorname{dim}(Z)$-dimensional complex vector space. If $E$ is as above then $\mathrm{H}_{1}(Z, \mathbf{C})$ carries the natural structure of a free $E_{\mathbf{C}}:=E \otimes_{\mathbf{Q}} \mathbf{C}$-module of rank $d(Z, E)$. We have

$$
\mathrm{H}_{1}(Z, \mathbf{C})=\bigoplus_{\sigma \in \Sigma_{E}} \mathbf{C}_{\sigma} \mathrm{H}_{1}(Z, \mathbf{C})=\oplus_{\sigma \in \Sigma_{E}} \mathrm{H}_{1}(Z, \mathbf{Q})_{\sigma}
$$

where

$\mathrm{H}_{1}(Z, \mathbf{Q})_{\sigma}:=\mathbf{C}_{\sigma} \mathrm{H}_{1}(Z, \mathbf{C})=\left\{x \in \mathrm{H}_{1}(Z, \mathbf{C}) \mid e x=\sigma(e) x \quad \forall e \in E\right\}=\mathrm{H}_{1}(Z, \mathbf{Q}) \otimes_{E, \sigma} \mathbf{C}$.

Clearly, every $\mathrm{H}_{1}(Z, \mathbf{Q})_{\sigma}$ is a $d(Z, E)$-dimensional $\mathbf{C}$-vector subspace that is also a $E_{\mathbf{C}}$-submodule of $\mathrm{H}_{1}(Z, \mathbf{C})$.

There is a canonical Hodge decomposition ([3, chapter 1], [1, pp. 52-53])

$$
\mathrm{H}_{1}(Z, \mathbf{C})=H^{-1,0} \oplus H^{0,-1}
$$

where $H^{-1,0}=H^{-1,0}(Z)$ and $H^{0,-1}=H^{0,-1}(Z)$ are mutually "complex conjugate" $\operatorname{dim}(Z)$-dimensional complex vector spaces. This splitting is $\operatorname{End}^{0}(Z)$-invariant and the $\operatorname{End}^{0}(Z)$-module $H^{-1,0}$ is canonically isomorphic to the commutative Lie algebra $\operatorname{Lie}(Z)$ of $Z$. This implies that $H^{-1,0}$ and $\operatorname{Lie}(Z)$ are isomorphic as $E$-modules and even as $E_{\mathbf{C}}$-modules.

Let

$$
\mathfrak{f}_{H}^{0}=\mathfrak{f}_{H, Z}^{0}: \mathrm{H}_{1}(Z, \mathbf{C}) \rightarrow \mathrm{H}_{1}(Z, \mathbf{C})
$$

be the $\mathbf{C}$-linear operator in $\mathrm{H}_{1}(Z, \mathbf{C})$ defined as follows.

$$
\mathfrak{f}_{H}(x)=-\frac{1}{2} x \quad \forall x \in H^{-1,0} ; \quad \mathfrak{f}_{H}^{0}(x)=\frac{1}{2} x \quad \forall x \in H^{0,-1} .
$$

Clearly, $\mathfrak{f}_{H}^{0}$ commutes with $\operatorname{End}^{0}(Z)$. In particular, every $\mathrm{H}_{1}(Z, \mathbf{Q})_{\sigma}$ is $\mathfrak{f}_{H}^{0}$-invariant. More precisely, the linear operator $\mathfrak{f}_{H}^{0}: \mathrm{H}_{1}(Z, \mathbf{Q})_{\sigma} \rightarrow \mathrm{H}_{1}(Z, \mathbf{Q})_{\sigma}$ is semisimple and its spectrum lies in the two-element set $\{1 / 2,-1 / 2\}$. Taking into account that the $E_{\mathbf{C}}$-modules $H^{-1,0}$ and $\operatorname{Lie}(Z)$ are isomorphic, we conclude that the multiplicity of eigenvalue $-1 / 2$ is $n_{\sigma}=n_{\sigma}(Z, E)$ while the multiplicity of eigenvalue $1 / 2$ is $d(Z, E)-$ $n_{\sigma}(Z, E)$. Let $\bar{\sigma}: E \hookrightarrow \mathbf{C}$ be the composition of $\sigma: E \hookrightarrow \mathbf{C}$ and the complex conjugation $\mathbf{C} \rightarrow \mathbf{C}$. It is known ([1], [2]) that

$$
n_{\sigma}+n_{\bar{\sigma}}=d(Z, E) \text {. }
$$

This implies that the multiplicity of eigenvalue $1 / 2$ is $n_{\bar{\sigma}}$.

We refer to [5], [10, Sect. 6.6.1 and 6.6.2] for the definition and basic properties of the Hodge group (aka special Mumford-Tate group) $\mathrm{Hdg}=\mathrm{Hdg}_{Z}$ of (the rational Hodge structure $\mathrm{H}_{1}(Z, \mathbf{Q})$ and of) $Z$. Recall that Hdg is a connected reductive algebraic Q-subgroup of $\mathrm{GL}\left(\mathrm{H}_{1}(Z, \mathbf{Q})\right)$, whose centralizer in $\operatorname{End}_{\mathbf{Q}}\left(\mathrm{H}_{1}(Z, \mathbf{Q})\right)$ coincides with $\operatorname{End}^{0}(Z)$. Let

$$
\operatorname{hdg}=\operatorname{hdg}_{Z} \subset \operatorname{End}_{\mathbf{Q}}\left(\mathrm{H}_{1}(Z, \mathbf{Q})\right)
$$


be the Q-Lie algebra of $\mathrm{Hdg}$; it is a reductive $\mathbf{Q}$-Lie subalgebra of $\operatorname{End}_{\mathbf{Q}}\left(\mathrm{H}_{1}(Z, \mathbf{Q})\right)$, its natural representation in $\mathrm{H}_{1}(Z, \mathbf{Q})$ is completely reducible and its centralizer there coincides with $\operatorname{End}^{0}(Z)$. Notice also that its complexification

$$
\operatorname{hdg}_{\mathbf{C}}=\operatorname{hdg} \otimes_{\mathbf{Q}} \mathbf{C} \subset \operatorname{End}_{\mathbf{Q}}\left(\mathrm{H}_{1}(Z, \mathbf{Q})\right) \otimes_{\mathbf{Q}} \mathbf{C}=\operatorname{End}_{\mathbf{C}}\left(\mathrm{H}_{1}(Z, \mathbf{C})\right)
$$

contains $\mathfrak{f}_{H}^{0}[9$, Sect. 3.4].

Suppose that $E=\operatorname{End}^{0}(Z)$ is a CM field. Choose a polarization on $Z$. The corresponding Rosati involution on $\operatorname{End}^{0}(Z)$ coincides with the complex conjugation $e \mapsto \bar{e}$ on $E$. The polarization gives rise to the nondegenerate alternating $\mathbf{Q}$-bilinear form

$$
\psi: \mathrm{H}_{1}(Z, \mathbf{Q}) \times \mathrm{H}_{1}(Z, \mathbf{Q}) \rightarrow \mathbf{Q}
$$

such that

$$
\psi(e x, y)=\psi(x, \bar{e} y) \forall x, y \in \mathrm{H}_{1}(Z, \mathbf{Q}) ; e \in E .
$$

Let

$$
E^{+}=\{e \in E \mid \bar{e}=e\}
$$

be the maximal totally real subfield of the CM field $E$ and let

$$
E_{-}=\{e \in E \mid \bar{e}=-e\} .
$$

Pick a non-zero element $\alpha \in \mathbf{Q}\left(\zeta_{q}\right)_{-}$. Now the standard construction (see, for instance, [5, p. 531]) allows us to define the non-degenerate $E$-sesquilinear Hermitian form

$$
\phi: \mathrm{H}_{1}(Z, \mathbf{Q}) \times \mathrm{H}_{1}(Z, \mathbf{Q}) \rightarrow E
$$

such that

$$
\psi(x, y)=\operatorname{Tr}_{E / \mathbf{Q}}(\alpha \phi(x, y)) \forall x, y \in \mathrm{H}_{1}(Z, \mathbf{Q}) .
$$

We write $\mathrm{U}\left(\mathrm{H}_{1}(Z, \mathbf{Q}), \phi\right)$ for the unitary group of $\phi$ of the $E$-vector space $\mathrm{H}_{1}(Z, \mathbf{Q})$, viewed as an algebraic Q-subgroup of $\mathrm{GL}\left(\mathrm{H}_{1}(Z, \mathbf{Q})\right.$ ) (via Weil's restriction of scalars from $E^{+}$to $\mathbf{Q}$ (ibid). It is well-known that $\mathrm{U}\left(\mathrm{H}_{1}(Z, \mathbf{Q}), \phi\right)$ is reductive and its $\mathbf{Q}$ dimension is

$$
\left[E^{+}: \mathbf{Q}\right] d(Z, E)^{2}=\frac{1}{2}[E: \mathbf{Q}] d(Z, E)^{2} .
$$

Let $\mathfrak{u}\left(\mathrm{H}_{1}(Z, \mathbf{Q}), \phi\right)$ be the $\mathbf{Q}$-Lie algebra of $\mathrm{U}\left(\mathrm{H}_{1}(Z, \mathbf{Q}), \phi\right)$ : it is a reductive $\mathbf{Q}$-Lie subalgebra of $\operatorname{End}_{\mathbf{Q}}\left(\mathrm{H}_{1}(Z, \mathbf{Q})\right)$. Explicitly,

$\mathfrak{u}\left(\mathrm{H}_{1}(Z, \mathbf{Q}), \phi\right)=\left\{u \in \operatorname{End}_{E}\left(\mathrm{H}_{1}(Z, \mathbf{Q})\right) \mid \phi(u x, y)+\overline{\phi(x, u y)}=0 \forall x, y \in \mathrm{H}_{1}(Z, \mathbf{Q})\right\}$.

The reductive Q-Lie algebra $\mathfrak{u}\left(\mathrm{H}_{1}(Z, \mathbf{Q}), \phi\right)$ splits into a direct sum

$$
\mathfrak{u}\left(\mathrm{H}_{1}(Z, \mathbf{Q}), \phi\right)=E_{-} \oplus \mathfrak{s} \mathfrak{u}\left(\mathrm{H}_{1}(Z, \mathbf{Q}), \phi\right)
$$

of its center $E_{-}$and the semisimple $\mathbf{Q}$-Lie algebra

$$
\mathfrak{s u}\left(\mathrm{H}_{1}(Z, \mathbf{Q}), \phi\right)=\left\{u \in \mathfrak{u}\left(\mathrm{H}_{1}(Z, \mathbf{Q}), \phi\right) \mid \operatorname{Tr}_{E}(u)=0 .\right\}
$$

Here

$$
\operatorname{Tr}_{E}: \operatorname{End}_{E}\left(\mathrm{H}_{1}(Z, \mathbf{Q})\right) \rightarrow E
$$

is the trace map. One may easily check that

$$
\operatorname{dim}_{\mathbf{Q}}\left(\mathfrak{s u}\left(\mathrm{H}_{1}(Z, \mathbf{Q}), \phi\right)\right)=\frac{1}{2}[E: \mathbf{Q}]\left\{d(Z, E)^{2}-1\right\} .
$$


Since the Hodge group respects the polarization and commutes with endomorphisms of $Z$,

$$
\operatorname{Hdg}(Z) \subset \mathrm{U}\left(\mathrm{H}_{1}(Z, \mathbf{Q}), \phi\right)
$$

(ibid). This implies that

$$
\operatorname{hdg} \subset \mathfrak{u}\left(\mathrm{H}_{1}(Z, \mathbf{Q}), \phi\right) \subset \operatorname{End}_{\mathbf{Q}}\left(\mathrm{H}_{1}(Z, \mathbf{Q})\right) .
$$

This implies that the semisimple part hdg ${ }^{s s}=[\mathrm{hdg}, \mathrm{hdg}]$ of hdg lies in $\mathfrak{s u}\left(\mathrm{H}_{1}(Z, \mathbf{Q}), \phi\right)$. In particular,

$$
\operatorname{dim}_{\mathbf{Q}}\left(\operatorname{hdg}^{s s}\right) \leq \frac{1}{2}[E: \mathbf{Q}]\left\{d(Z, E)^{2}-1\right\}
$$

the equality holds if and only if $\mathrm{hdg}^{s s}=\mathfrak{s u}\left(\mathrm{H}_{1}(Z, \mathbf{Q}), \phi\right)$.

The following statement may be viewed as a partial generalization of Theorem 3 in $[5$, p. 526].

Theorem 1.1. Suppose that $E=\operatorname{End}^{0}(Z)$ is a $C M$ field. Assume that all $n_{\sigma}(Z, E)$ are distinct positive integers. Assume additionally that there exists a field embedding $\sigma: E \hookrightarrow \mathbf{C}$ such that $n_{\sigma}(Z, E)$ and $d(Z, E)$ are relatively prime.

Then $\mathrm{hdg}^{s s}=\mathfrak{s u}\left(\mathrm{H}_{1}(Z, \mathbf{Q}), \phi\right)$.

Remark 1.2. Clearly, in the course of the proof of Theorem 1.1, it suffices to check that

$$
\operatorname{dim}_{\mathbf{Q}}\left(\mathrm{hdg}^{s s}\right) \geq \frac{1}{2}[E: \mathbf{Q}]\left\{d(Z, E)^{2}-1\right\} .
$$

We prove Theorem 1.1 in Section 4.

Proof of Theorem 0.1. Let us put $Z=J^{(f, q)}$ and $E=\mathbf{Q}\left(\zeta_{q}\right)$. Clearly,

$$
d(Z, E)=n-1 \text {. }
$$

We know that $\operatorname{End}^{0}(Z)=E=\mathbf{Q}\left(\zeta_{q}\right)[16,18]$ and if $\sigma=\sigma_{i}: \mathbf{Q}\left(\zeta_{q}\right) \hookrightarrow \mathbf{C}$ is a field embedding that sends $\zeta_{q}$ to $\zeta_{q}^{-i}$ with $1 \leq i<q,(i, p)=1$ then $n_{\sigma}=[n i / q][16,17]$. (Clearly, every field embedding $\mathbf{Q}\left(\zeta_{q}\right) \hookrightarrow \mathbf{C}$ is of the form $\sigma_{i}$.) Since $n>q$, all integers $[n i / q]$ are positive and distinct. Propositions 2.1 and 2.2 below imply that under our assumptions on $(p, q, n)$ there exists a positive integer $i<q$ such that $(i, p)=1$ and $[n i / q]$ and $n-1$ are relatively prime. This allows us to apply Theorem 1.1 and conclude that $\mathrm{hdg}^{s s}=\mathfrak{s u}\left(\mathrm{H}_{1}(Z, \mathbf{Q}), \phi\right)$. On the other hand, by a result from [9], the center of hdg coincides with $E_{-}$. This implies that the reductive Q-Lie algebra hdg coincides with the direct sum

$$
E_{-} \oplus \mathfrak{s u}\left(\mathrm{H}_{1}(Z, \mathbf{Q}), \phi\right)=\mathfrak{u}\left(\mathrm{H}_{1}(Z, \mathbf{Q}), \phi\right) .
$$

Now the connectedness of the Hodge group and the unitary group implies that $\operatorname{Hdg}(Z)=\mathrm{U}\left(\mathrm{H}_{1}(Z, \mathbf{Q}), \phi\right)$, i.e.,

$$
\operatorname{Hdg}\left(J^{(f, q)}\right)=\mathrm{U}\left(\mathrm{H}_{1}\left(J^{(f, q)}, \mathbf{Q}\right), \phi_{q}\right) .
$$




\section{Divisibility properties of integral parts}

Proposition 2.1. Suppose that $p$ is a prime, $r$ a positive integer, $q=p^{r}$ and $n$ is a positive integer that is not divisible by $p$.

Suppose that one of the following conditions holds:

(i) $q<n<2 q$;

(ii) The prime $p$ is odd. In addition, either $p \nmid(n-1)$ or $n<2 q$.

Then there exists an integer $i$ such that

$$
1 \leq i \leq q-1,(i, p)=1
$$

and integers $[n i / p]$ and $n-1$ are relatively prime.

Proof. If $q<n<2 q$ then

$$
[n \cdot 1 / q]=[n / q]=1
$$

and we may take $i=1$.

So, further we assume that $p$ is odd and either $n<q$ or $n-1$ is not divisible by $p$.

If $q>n>q / 2$ then $[2 n / q]=1$ and we may take $i=2$.

If $0<n<q / 2$ then there exists a positive integer $\mu$ such that

$$
q \leq \mu n<(\mu+1) n<2 q .
$$

Since $q$ is a power of $p$ and $n$ is not divisible by $p$,

$$
q<\mu n<(\mu+1) n<2 q .
$$

Clearly,

$$
1=[n \mu / q]=[n(\mu+1) / q] .
$$

So, we take as $i$ either $\mu$ or $\mu+1$, depending on which one is not divisible by $p$.

Now let us assume that $n-1$ is not divisible by $q$. Let us put

$$
k=[n / q], c=n-k q, d=c-1 .
$$

We have

$$
\begin{gathered}
c=d+1, n=q k+c, n-1=q k+d ; 2 \leq c \leq q-1,1 \leq d \leq q-2, \\
(c, p)=1,(d, p)=1 .
\end{gathered}
$$

Let $i$ be an integer such that $1 \leq i \leq q-1$ and $(i, p)=1$. Put $j=[c i / q]$. Clearly, $j$ is a nonnegative integer such that

$$
q j<i c<(q+1) j .
$$

(The first inequality holds, because neither $c$ nor $i$ are divisible by $p$.) In other words,

$$
0<i c-q j<q .
$$

In addition,

$$
[n i / q]=[(k q+c) i / q]=i k+[c i / q]=i k+j .
$$

Suppose that $n-1$ and $[n i / q]$ are not relatively prime. Then there exists a prime $\ell$ that divides both $n-1$ and $[n i / q]$. This implies that $q \cdot k+d \cdot 1=0$ in $\mathbf{Z} / \ell \mathbf{Z}$ and $i \cdot k+j \cdot 1=0$ in $\mathbf{Z} / \ell \mathbf{Z}$. So, we get the homogeneous system of two linear equations over the field $\mathbf{Z} / \ell \mathbf{Z}$ that admits the nontrivial solution $(k, 1) \neq(0,0)$. By Cramer's rule, the determinant $i d-q j$ is zero in $\mathbf{Z} / \ell \mathbf{Z}$, i.e., the integer $i d-q j$ is divisible by 
$\ell$. In particular, $i d-q j \neq \pm 1$. So, we prove the Proposition if we find such $i$ that $i d-q j$ is either 1 or -1 . In order to do that, notice that

$$
i d-q j=i(c-1)-q j=(i c-q j)-i \text {. }
$$

This implies that

$$
-i<i d-q j<q-i
$$

Since $1 \leq i \leq q-1$,

$$
1-q<i d-q j<q-1 .
$$

Now if we choose $i$ in such a way that $1 \leq i \leq q-1$ and $i d$ is congruent to 1 modulo $q$ (such choice is possible, because $d$ is not divisible by $p$ ) then $i d-q j$ is congruent to 1 modulo $q$ and therefore $i d-q j=1$. In addition, the latter equality implies that $i$ is not divisible by $p$.

Proposition 2.1 admits the following (partial) generalization.

Proposition 2.2. Suppose that $p$ is a prime, $r$ a positive integer, $q=p^{r}$ and $n$ is a positive integer that is not divisible by $p$. Suppose that $n-1$ is not divisible by $q$. If $p=2$, assume additionally that $q=2^{r}>2$ and $n \not \equiv q-1 \bmod 2 q$.

Then there exists an integer $i$ such that

$$
1 \leq i \leq q-1,(i, p)=1
$$

and integers $[n i / p]$ and $n-1$ are relatively prime.

Remark 2.3. If $q=p=2$ then every odd $n$ is congruent to 1 modulo 2 .

If $p=2, q=4$ then $(n, q)$ satisfy the conditions of Proposition 2.2 if and only if $n-7$ is divisible by 8 .

Proof of Proposition 2.2. As in the proof of Proposition 2.1, let us put

$$
k=[n / q], \quad c=n-k q, \quad d=c-1 .
$$

We have

$$
(c, p)=1, \quad 2 \leq c \leq q-1, \quad 1 \leq d \leq q-2 .
$$

We are given that $q$ does not divide $d$. However, in the light of Proposition 2.1, we may and will assume that $p$ divides $d$; in particular, $q>p$ and $d \geq p \geq 2>1$.

Let

Then

$$
t=(d, q), \quad d^{\prime}=d / t, \quad q^{\prime}=q / t
$$

$$
q^{\prime}>1, t>1,\left(d^{\prime}, q^{\prime}\right)=1
$$

and both $t$ and $q^{\prime}$ are powers of $p$. This implies that

$$
\left(d^{\prime}, p\right)=1, t \geq p \geq 2, q^{\prime} \geq p \geq 2>1 \text {. }
$$

Since $q^{\prime}>1, d^{\prime} \geq 1$ and $\left(d^{\prime}, q^{\prime}\right)=1$, there exists a unique pair of integers $(i, j)$ such that

$$
d^{\prime} i-q^{\prime} j=1, \quad 0<i \leq q^{\prime}-1, \quad j \geq 0 .
$$

Clearly, $(i, p)=1$ and therefore $\left(i+q^{\prime}, p\right)=1$, because $q^{\prime}$ is a power of $p$. Since $t \geq 2$, we have $i+q^{\prime}<2 q^{\prime} \leq t q^{\prime}=q$.

We will treat the case $p=2, n \equiv-1 \bmod q$ and $n \not \equiv q-1 \bmod 2 q$ separately at the end. So we further assume that if $p=2$, then $n+1$ is not divisible by $q$. We 
will show that either $i$ or $i+q^{\prime}$ is the desired integer, i.e., either $[n i / q]$ and $n-1$ are relatively prime or $\left[n\left(i+q^{\prime}\right) / q\right]$ and $n-1$ are relatively prime. It is convenient to consider both integers $[n i / q]$ and $\left[n\left(i+q^{\prime}\right) / q\right]$ as $\left[n\left(i+\epsilon q^{\prime}\right) / q\right]$ with $\epsilon=0,1$.

For every nonnegative integer $\epsilon$ we have

$$
d^{\prime}\left(i+\epsilon q^{\prime}\right)-q^{\prime}\left(j+\epsilon d^{\prime}\right)=1 .
$$

Multiplying both side of (2) by $t$, we get

$$
d\left(i+\epsilon q^{\prime}\right)-q\left(j+\epsilon d^{\prime}\right)=t .
$$

Since $c=d+1$, it follows that

$$
\frac{c\left(i+\epsilon q^{\prime}\right)}{q}=\frac{(d+1)\left(i+\epsilon q^{\prime}\right)}{q}=j+\epsilon d^{\prime}+\frac{t+i+\epsilon q^{\prime}}{q}
$$

and therefore

$$
\left[\frac{c\left(i+\epsilon q^{\prime}\right)}{q}\right]-\left(j+\epsilon d^{\prime}\right)=\left[\frac{t+i+\epsilon q^{\prime}}{q}\right] .
$$

The following Lemma will be proven at the end of this Section.

Lemma 2.4. We keep the assumptions of Proposition 2.2. If $p=2$, we assume additionally that $q \nmid n+1$. Then $\left[\left(t+i+q^{\prime}\right) / q\right]=0$ and therefore $[(t+i) / q]=0$.

Let us assume that either $p$ is odd or $p=2$ and $n+1$ is not divisible by $q$. Combining Lemma 2.4 with (4), we conclude that

$$
\left[\frac{c\left(i+\epsilon q^{\prime}\right)}{q}\right]=\left(j+\epsilon d^{\prime}\right)
$$

if $\epsilon=0$ or 1. It follows that if $\epsilon=0$ or 1 then

$\left[n\left(i+\epsilon q^{\prime}\right) / q\right]=\left[(k q+c)\left(i+\epsilon q^{\prime}\right) / q\right]=k\left(i+\epsilon q^{\prime}\right)+\left[c\left(i+\epsilon q^{\prime}\right) / q\right]=k\left(i+\epsilon q^{\prime}\right)+\left(j+\epsilon d^{\prime}\right)$.

Now suppose that $n-1$ and $\left.\left[n\left(i+\epsilon q^{\prime}\right)\right] / q\right]$ are not relatively prime for some $\epsilon=0$ or 1 . Then there exists a prime $\ell$ that divides both $n-1$ and $\left[n\left(i+\epsilon q^{\prime}\right) / q\right]$. This implies that $q \cdot k+d \cdot 1=0$ in $\mathbf{Z} / \ell \mathbf{Z}$ and $\left(i+\epsilon q^{\prime}\right) \cdot k+\left(j+\epsilon d^{\prime}\right) \cdot 1=0$ in $\mathbf{Z} / \ell \mathbf{Z}$. So we get the homogeneous system of linear equations over the field $\mathbf{Z} / \ell \mathbf{Z}$ with determinant

$$
d\left(i+\epsilon q^{\prime}\right)-q\left(j+\epsilon d^{\prime}\right)=t
$$

(by $(3)$ ), which admits a non-trivial solution $(k, 1) \neq(0,0)$. By Cramer's rule the determinant $t$ is zero in $\mathbf{Z} / \ell \mathbf{Z}$, i.e., $\ell \mid t$. Since $t$ is a power of $p$, we conclude that $\ell=p$.

Since $\left(i+\epsilon q^{\prime}\right) \cdot k+\left(j+\epsilon d^{\prime}\right) \cdot 1=0$ in $\mathbf{Z} / \ell \mathbf{Z}$ and $\ell=p$, the integer $\left(i+\epsilon q^{\prime}\right) \cdot k+\left(j+\epsilon d^{\prime}\right)$ is divisible by $p$.

Now suppose that $n-1$ and $\left[n\left(i+\epsilon q^{\prime}\right) / q\right]$ are not relatively prime for both $\epsilon=0$ and 1 . This implies that both integers $i \cdot k+j$ and $\left(i+q^{\prime}\right) \cdot k+\left(j+d^{\prime}\right)$ are divisible by $p$. Therefore their difference $q^{\prime} k+d^{\prime}$ is also divisible by $p$, which is not the case, because $q^{\prime}$ is a power of $p$ while $\left(d^{\prime}, p\right)=1$. The obtained contradiction proves Proposition when either $p$ is odd or $p=2$ and $n+1$ is not divisible by $q$.

At last, let us treat the remaining case when $p=2, q=2^{r}$ with $r \geq 2$, the integer $n+1$ is divisible by $q$ but $n \not \equiv q-1 \bmod 2 q$. Then $q$ divides $n+1$ and the ratio 
$k:=(n+1) / q$ is an even integer. We have $n=2^{r} k-1$. Let us put $i=2^{r-1}-1$. Since $r \geq 2$, the integer $i$ is odd. We have

$$
\left[\frac{n i}{q}\right]=\left[\frac{\left(2^{r} k-1\right)\left(2^{r-1}-1\right)}{2^{r}}\right]=\left(2^{r-1}-1\right) k-1 .
$$

It follows that

$$
[n i / q] \equiv-1 \quad \bmod k .
$$

In particular, $[n i / q]$ is odd, since $k$ is even. Notice that

$$
(n-1) / 2=[n i / q]+k .
$$

Combining all those assertions, we get

$$
\begin{aligned}
(n-1,[n i / q]) & =((n-1) / 2,[n i / q])=([n i / q]+k,[n i / q]) \\
& =(k,[n i / q])=(k,-1)=1 .
\end{aligned}
$$

Proof of Lemma 2.4. Recall that $q^{\prime}$ and $t$ are powers of $p$. This implies that $t \geq p \geq 3$ if $p$ is odd; if $p=2$ then either $t=2$ or $t \geq 4$.

First, let us assume that $p$ is odd and therefore $t \geq p \geq 3$ and $q^{\prime} \geq p \geq 3$. It follows that

$$
q^{\prime}(t-2) \geq 3(t-2)=3 t-6 \geq t .
$$

Since $i \leq q^{\prime}-1$,

$$
q=t q^{\prime} \geq t+2 q^{\prime}>t+i+q^{\prime} .
$$

This implies that $\left[\left(t+i+q^{\prime}\right) / q\right]=0$.

Second, assume that $p=2$ and $t \geq 4$. Then $q^{\prime} \geq 2$ and therefore

$$
q^{\prime}(t-2) \geq 2(t-2)=2 t-4 \geq t .
$$

As above, $i \leq q^{\prime}-1$,

$$
q=t q^{\prime}=(t-2) q^{\prime}+2 q^{\prime} \geq t+2 q^{\prime}>t+i+q^{\prime}
$$

and therefore $\left[\left(t+i+q^{\prime}\right) / q\right]=0$.

Third, assume that $p=2$ and $t=2$. Then

$$
t=2, d=2 d^{\prime}, q^{\prime}=\frac{q}{2}=2^{r-1} .
$$

Recall that $(i, p)=1$, i. e., $i$ is odd. Since $i \leq q^{\prime}-1$, the sum

$$
t+i+q^{\prime}=2+i+2^{r-1}
$$

is greater or equal than $q=2^{r}$ only if $i=q^{\prime}-1=2^{r-1}-1$. By (2), $d^{\prime} \cdot i \equiv 1 \bmod q^{\prime}$. If $i=q^{\prime}-1$, then $d^{\prime}=q^{\prime}-1$, since $1 \leq d^{\prime}<q^{\prime}$. This implies that

$$
c=d+1=2 d^{\prime}+1=2 q^{\prime}-1=q-1,
$$

which contradicts the assumption that

$$
n+1=(k q+c)+1=k q+(c+1)
$$

is not divisible by $q$. So, this case does not occur. 


\section{Linear reductive Lie algebras}

Throughout this Section, $Q$ is a field of characteristic zero, $C$ is an algebraically closed field containing $Q$. If $W$ is a $Q$-vector space (resp. $Q$-algebra or $Q$-Lie algebra) then we write $W_{C}$ for $W \otimes_{Q} C$ provided with the natural structure of a $C$-vector space (resp. $C$-algebra or $C$-Lie algebra).

Let $W$ be a nonzero finite-dimensional $Q$-vector space. Let $E \subset \operatorname{End}_{Q}(W)$ be a subfield that contains the scalars $Q \cdot \operatorname{Id}_{W}$. Then $E / Q$ is a finite algebraic extension and $W$ carries the natural structure of $E$-vector space; in addition,

$$
\operatorname{dim}_{Q}(W)=[E: Q] \cdot \operatorname{dim}_{E}(W) .
$$

We write $\Sigma$ for the set of all $Q$-linear field embedding $\sigma: E \hookrightarrow C$. If $\sigma \in \Sigma$ then we write $W_{\sigma}$ for the $C$-vector space $W \otimes_{E, \sigma} C$; clearly,

$$
\operatorname{dim}_{C}\left(W_{\sigma}\right)=\operatorname{dim}_{E}(W)
$$

there are natural surjective homomorphisms $W_{C} \rightarrow W_{\sigma}$. Their "direct sum"

$$
W_{C} \rightarrow \oplus_{\sigma \in \Sigma} W_{\sigma}
$$

is an isomorphism of $C$-vector spaces, so one may view every $W_{\sigma}$ as a $C$-vector subspace (direct summand) of $W_{C}$.

Remark 3.1. Let $S$ be a $Q$-vector subspace of $W$. Let $E S \subset W$ be the $E$-vector subspace of $W$ generated by $S$, i.e., $E S$ is the set of all linear combinations of elements of $S$ with coefficients in $E$. Clearly, $E S=W$ if and only if $S$ contains a basis of the $E$-vector space $W$. It is also clear that the image of the composition

$$
S_{C} \subset W_{C} \rightarrow W_{\sigma}
$$

coincides with

$$
\{E S\}_{\sigma}=E S \otimes_{E, \sigma} C \subset W \otimes_{E, \sigma} C=W_{\sigma} ;
$$

in particular, the $C$-dimension of this image coincides with $\operatorname{dim}_{E}(E S)$ and does not depend on the choice of $\sigma$.

Remark 3.2. Let $\mathfrak{k} \subset \operatorname{End}_{Q}(W)$ be a reductive $Q$-Lie subalgebra such that the natural representation of $\mathfrak{k}$ is completely reducible and the centralizer $\operatorname{End}_{\mathfrak{k}}(W)$ of $\mathfrak{k}$ in $\operatorname{End}_{Q}(W)$ coincides with $E$; in particular, the $\mathfrak{k}$-module $W$ is simple and $\mathfrak{k} \subset$ $\operatorname{End}_{E}(W)$. Clearly, $E \mathfrak{k}$ is a reductive $E$-Lie subalgebra of $\operatorname{End}_{E}(W)$ and the centralizer of $E \mathfrak{k}$ in $\operatorname{End}_{E}(W)$ coincides with $E$. In other words, the $E$-vector space $W$ is an absolutely simple $E \mathfrak{k}$-module. This implies that the $C$-vector space $W_{\sigma}$ is an absolutely simple $\{E \mathfrak{k}\}_{\sigma}$-module. However, applying Remark 3.1 to the $E$-vector space $\operatorname{End}_{E}(W)$ (instead of $W$ ) and its $Q$-vector subspace $S=\mathfrak{k}$, we conclude that the $C$-Lie subalgebra $\{E \mathfrak{k}\}_{\sigma} \subset \operatorname{End}_{C}\left(W_{\sigma}\right)$ is the image of

$$
\mathfrak{k}_{C} \rightarrow \operatorname{End}_{E}(W) \otimes_{E, \sigma} C=\operatorname{End}_{C}\left(W_{\sigma}\right) .
$$

This implies that the $\mathfrak{k}_{C}$-module $W_{\sigma}$ is also absolutely simple. On the other hand, the reductiveness of $\mathfrak{k}$ and the equality $\operatorname{End}_{\mathfrak{k}}(W)=E$ imply that $\mathfrak{k}$ splits into the direct sum

$$
\mathfrak{k}=\mathfrak{k}^{s s} \oplus \mathfrak{c}
$$

of its center $\mathfrak{c}$ and the semisimple $Q$-Lie algebra $\mathfrak{k}^{s s}=[\mathfrak{k}, \mathfrak{k}]$; in addition, $\mathfrak{c} \subset E$. This implies that $E \mathfrak{k}=E \mathfrak{k}^{\mathfrak{s s}} \oplus E \mathfrak{c}$ where $E \mathfrak{c}$ is either $E$ or $\{0\}$. It follows easily that in both 
cases the $\mathfrak{k}_{C}^{s s}$-module $W_{\sigma}$ remains absolutely simple and the image of $\mathfrak{k}_{C}^{s s}$ in $\operatorname{End}_{C}\left(W_{\sigma}\right)$ coincides with

$$
\left\{E \mathfrak{k}^{s s}\right\}_{\sigma}=E \mathfrak{k}^{s s} \otimes_{E, \sigma} C=\left[\{E \mathfrak{k}\}_{\sigma},\{E \mathfrak{k}\}_{\sigma}\right] .
$$

The following statement is a variant of [6, Sect. 4, Prop. 5]

Lemma 3.3. Let $W$ be a finite-dimensional $C$-vector space of positive dimension, let $\mathfrak{s}(W)$ be the Lie algebra of traceless linear operators in $W$. Let $\mathfrak{g} \subset \operatorname{End}_{C}(W)$ be an irreducible semisimple linear C-Lie subalgebra. Assume that there exists a diagonalizable operator

$$
f \in \mathfrak{g} \subset \operatorname{End}_{C}(W)
$$

that enjoys the following property: $f$ acts in $W$ as a linear operator with exactly two eigenvalues, whose multiplicities are relatively prime.

Then $\mathfrak{g}=\mathfrak{s l}(W)$.

Proof of Lemma 3.3. Clearly, $f \neq 0$ (otherwise, it would have only one eigenvalue).

Let $\alpha_{1}$ and $\alpha_{2}$ be the eigenvalues of $f$ and let $W_{1}$ and $W_{2}$ are the corresponding eigenspaces. Since $f \neq 0$, either $\alpha_{1} \neq 0$ or $\alpha_{2} \neq 0$. We claim that $\mathfrak{g}$ is simple. Indeed, let us split the semisimple $\mathfrak{g}$ into a direct sum $\mathfrak{g}=\oplus_{i=1}^{r} \mathfrak{g}_{i}$ of simple $C$-Lie algebras $\mathfrak{g}_{i}$. Then the simple $\mathfrak{g}$-module $W$ splits into a tensor product $W=\otimes_{i=1}^{r} U_{i}$ of simple $\mathfrak{g}_{i}$ modules $U_{i}$. Since $W$ is a faithful $\mathfrak{g}$-module, all $\operatorname{dim}_{C}\left(U_{i}\right)>1$. We have $f=\sum_{i=1}^{r} f_{i}$ with $f_{i} \in \mathfrak{g}_{i}$. Clearly, if some $f_{i}=0$ then the multiplicities of all eigenvalues of $f$ are divisible by $\operatorname{dim}_{C}\left(U_{i}\right)$. However, the spectrum of $f$ consists of (only) two eigenvalues, whose multiplicities are relatively prime. This implies that all $f_{i} \neq 0$, i.e., $f$ does not belong to a proper ideal of $\mathfrak{g}$. Now the simplicity of $\mathfrak{g}$ follows from [11, Th. 1.5 on p. 286] (with $k=C$ and $V=W$ ).

The semisimplicity of $f$ means that

$$
W=W_{1} \oplus W_{2} .
$$

Let us put

$$
n=\operatorname{dim}_{C}\left(W_{1}\right), m=\operatorname{dim}_{C}\left(W_{2}\right) .
$$

By assumption, $n$ and $m$ are positive integers that are relatively prime. Let us put

$$
h:=n+m=\operatorname{dim}_{C}(W) .
$$

Clearly, $h$ and $m$ are relatively prime.

The semisimplicity of $\mathfrak{g}$ implies that $\mathfrak{g} \subset \mathfrak{s l}(W)$; in particular, the trace of $f$ is zero. This means that

$$
n \alpha_{1}+m \alpha_{2}=0 .
$$

It follows that both $\alpha_{1}$ and $\alpha_{2}$ do not vanish. Replacing $f$ by $\left(m / \alpha_{1}\right) f$, we may and will assume that the spectrum of $f$ consists of eigenvalue $m$ of multiplicity $n$ and eigenvalue $-n$ of multiplicity $m$. In addition, $W_{1}$ is the eigenspace attached to eigenvalue $m$ and $W_{2}$ is the eigenspace attached to eigenvalue $-n$.

Let $\Theta \subset \mathrm{GL}(W)$ be the linear algebraic subgroup that consists of all linear operators $A(u, v)$ that act on $W_{1}$ as multiplication by $u$ and on $W_{2}$ as multiplication by $v$ where $(u, v) \in C^{*} \times C^{*}$ satisfy

$$
u^{n} v^{m}=1 .
$$


Let us consider the multiplicative algebraic group $\mathbf{G}_{m}$ over $C$. The morphism of algebraic $C$-groups

$$
\rho: \mathbf{G}_{m} \rightarrow \Theta, z \mapsto A\left(z^{m}, z^{-n}\right)
$$

is an isomorphism: in order to construct the inverse map, pick integers $a$ and $b$ with $m a-n b=1$ (here we use the assumption that $n$ and $m$ are relatively prime). Then the morphism of algebraic group

$$
\Theta \rightarrow \mathbf{G}_{m}, A(u, v) \mapsto u^{a} v^{b}
$$

is the inverse of $\rho$. So, $\Theta$ is isomorphic to $\mathbf{G}_{m}$; in particular, it is a one-dimensional connected linear algebraic group and its Lie algebra is a one-dimensional $C$-Lie subalgebra of $\operatorname{End}_{C}(W)$. Considering the tangent map to the composition

$$
\mathbf{G}_{m} \rightarrow \Theta \subset \mathrm{GL}(W),
$$

one may easily find that the Lie algebra $\operatorname{Lie}(\Theta)$ of $\Theta$ coincides with $C \cdot f \subset \operatorname{End}_{C}(W)$. On the other hand, let $\mu_{h} \subset C^{*}$ be the order $h$ cyclic group of $h$ th root of unity. If $z \in \mu_{h}$ then $z^{m}=z^{-n}$ (since $n+m=h$ ) and therefore $A\left(z^{m}, z^{-n}\right)$ coincides with multiplication by $z^{m}$. This implies that

$$
\mu_{h} \cdot \operatorname{Id} \subset \Theta \subset \mathrm{GL}(W)
$$

where Id is the identity map in $W$.

Since every (linear) simple Lie algebra is algebraic, there exists a connected simple linear algebraic subgroup $\mathfrak{G} \subset \mathrm{GL}(W)$, whose Lie algebra coincides with $\mathfrak{g}$. Since $f \in \mathfrak{g}$, we have $C \cdot f \subset \mathfrak{g}$. In other words, the Lie algebra of connected $\Theta$ lies in the Lie algebra of $\mathfrak{G}$. This implies that $\Theta \subset \mathfrak{G}$ and therefore

$$
\mu_{h} \cdot \text { Id } \subset \Theta \subset \mathfrak{G} \subset \mathrm{GL}(W) ;
$$

in particular, the order of the center of simple $\mathfrak{G}$ is divisible by $h=\operatorname{dim}_{C}(W)$. It follows from Lemma 2 of Sect. 4 in $[6]$ that $\mathfrak{G}=\mathrm{SL}(W)$. Since the Lie algebra of $\mathrm{SL}(W)$ coincides with $\mathfrak{s l}(W)$, we conclude that $\mathfrak{g}=\mathfrak{s l}(W)$.

Theorem 3.4. Let $V$ be a $Q$-vector space of positive finite dimension and let $\mathfrak{k} \subset$ $\operatorname{End}_{Q}(V)$ a reductive Lie algebra, whose natural representation in $V$ is completely reducible. Suppose that the centralizer

$$
E:=\operatorname{End}_{\mathfrak{k}}(V)
$$

is a field. Let us put

$$
h=\operatorname{dim}_{E}(V) .
$$

Let

$$
f \in \mathfrak{k}_{C} \subset \operatorname{End}_{Q}(V) \otimes_{Q} C=\operatorname{End}_{C}\left(V_{C}\right)
$$

be a non-zero semisimple element that enjoys the following properties.

(i) The spectrum of the $C$-linear operator $f: V_{C} \rightarrow V_{C}$ consists of two eigenvalues, $\lambda$ and $\mu$;

(ii) For every $\sigma \in \Sigma$ we write $n_{\sigma}$ and $m_{\sigma}$ for the multiplicities of of eigenvalues $\lambda$ and $\mu$ of the $C$-linear operator $f: V_{\sigma} \rightarrow V_{\sigma}$. Then:

(1) all the numbers $\left\{n_{\sigma}\right\}_{\sigma \in \Sigma}$ are distinct positive integers. Assume also that all $m_{\sigma}$ are positive integers.

(2) There exists $\tau \in \Sigma$ such that $n_{\tau}$ and $m_{\tau}$ are relatively prime. 
Then the semisimple part $\mathfrak{k}_{C}^{s s}=\left[\mathfrak{k}_{C}, \mathfrak{k}_{C}\right]$ of $\mathfrak{k}_{C}$ contains an ideal that is isomorphic to a direct sum of $r$ copies of the $C$-Lie algebra $\mathfrak{s l}(h, C)$ of traceless matrices of size $h$ with $r \geq[E: Q] / 2$. In particular, if $\mathfrak{k}^{s s}=[\mathfrak{k}, \mathfrak{k}]$ is the semisimple part of $\mathfrak{k}$ then

$$
\operatorname{dim}_{Q}\left(\mathfrak{k}^{s s}\right)=\operatorname{dim}_{C}\left(\mathfrak{k}_{C}^{s s}\right) \geq \frac{1}{2}[E: Q]\left(h^{2}-1\right) .
$$

In order to prove Theorem 3.4, we need the following two statements.

Lemma 3.5. Let $\mathfrak{g}_{1}$ and $\mathfrak{g}_{2}$ be non-zero finite-dimensional simple Lie algebras over a field of characteristic zero. Let $\mathfrak{g} \subset \mathfrak{g}_{1} \oplus \mathfrak{g}_{2}$ be a semisimple Lie subalgebra such that the both projection maps

$$
\mathfrak{g} \rightarrow \mathfrak{g}_{1}, \mathfrak{g} \rightarrow \mathfrak{g}_{2}
$$

are surjective. Then either $\mathfrak{g}=\mathfrak{g}_{1} \oplus \mathfrak{g}_{2}$ or there exists a Lie algebra isomorphism $\phi: \mathfrak{g}_{1} \cong \mathfrak{g}_{2}$ such that $\mathfrak{g}$ coincides with the graph $\Gamma(\phi)$ of $\phi$ in $\mathfrak{g}_{1} \times \mathfrak{g}_{2}=\mathfrak{g}_{1} \oplus \mathfrak{g}_{2}$.

In particular, if $\mathfrak{g}_{1}$ and $\mathfrak{g}_{2}$ are not isomorphic then $\mathfrak{g}=\mathfrak{g}_{1} \oplus \mathfrak{g}_{2}$.

Lemma 3.6. Let $n \geq 2$ and $d$ be positive integers. Let $a_{1}, \ldots, a_{d}$ be d distinct positive integers such that

$$
1 \leq a_{i}<n \forall i ; \quad a_{i} \neq n-a_{j} \forall i, j .
$$

Let $C$ be an algebraically closed field of characteristic zero and $W_{1}, \ldots, W_{d}$ be $n$ dimensional $C$-vector spaces. Let us put

$$
W=\oplus_{i=1}^{d} W_{i}
$$

and let

$$
\mathfrak{k}^{s s} \subset \oplus_{i=1}^{d} \mathfrak{s l}\left(W_{i}\right) \subset \oplus_{i=1}^{d} \operatorname{End}_{C}\left(W_{i}\right) \subset \operatorname{End}_{C}(W)
$$

be a semisimple $C$-Lie algebra that enjoys the following properties:

(i) The projection map $\mathfrak{k}^{s s} \rightarrow \mathfrak{s l}\left(W_{i}\right)$ is surjective for all $i$.

(ii) There exists a semisimple element

$$
f \in \mathfrak{k}^{s s} \subset \oplus_{i=1}^{d} \operatorname{End}_{C}\left(W_{i}\right)
$$

such that for all $i$ the element $f$ acts in $W_{i}$ as a linear operator with two eigenvalues of multiplicity $a_{i}$ and $n-a_{i}$ respectively.

Then $\mathfrak{k}^{s s}=\oplus_{i=1}^{d} \mathfrak{s} \mathfrak{l}\left(W_{i}\right)$.

Proof of Lemma 3.5. Let $\mathfrak{g}_{0}$ be the kernel of the first projection map $\mathfrak{g} \rightarrow \mathfrak{g}_{1}$. By definition,

$$
\mathfrak{g}_{0} \subset\{0\} \oplus \mathfrak{g}_{2} \subset \mathfrak{g}_{1} \oplus \mathfrak{g}_{2} .
$$

The surjectivity of the second projection $\mathfrak{g} \rightarrow \mathfrak{g}_{2}$ implies that $\mathfrak{g}_{0}$ is an ideal in $\{0\} \oplus$ $\mathfrak{g}_{2} \cong \mathfrak{g}_{2}$. The simplicity of $\mathfrak{g}_{2}$ implies that either $\mathfrak{g}_{0}=\{0\}$ or $\mathfrak{g}_{0}=\{0\} \oplus \mathfrak{g}_{2}$. In the latter case $\mathfrak{g}=\mathfrak{g}_{1} \oplus \mathfrak{g}_{2}$. So, let us assume that $\mathfrak{g}_{0}=\{0\}$, i.e., the first projection map is an isomorphism. This means that there is a Lie algebra homomorphism $\phi: \mathfrak{g}_{1} \rightarrow \mathfrak{g}_{2}$ such that $\mathfrak{g}$ coincides with the graph $\Gamma(\phi)$ of $\phi$. Now the surjectiveness of the second projection map means that $\phi$ is surjective. Since $\mathfrak{g}_{1}$ is simple, $\phi$ is injective and therefore is an isomorphism. 
Proof of Lemma 3.6. Let us denote by $f_{i}: W_{i} \rightarrow W_{i}$ the linear operator in $W_{i}$ induced by $f$ (for all $i$ ).

If $d=1$ then the result follows from the property (i). Assume now that $d=2$. If $\mathfrak{k}^{s s} \neq \mathfrak{s l}\left(W_{1}\right) \oplus \mathfrak{s l}\left(W_{2}\right)$ then it follows from Lemma 3.5 that $\mathfrak{k}^{s s}$ coincides with the graph $\Gamma(\phi)$ of a certain Lie algebra isomorphism $\phi: \mathfrak{s l}\left(W_{1}\right) \cong \mathfrak{s l}\left(W_{2}\right)$. It is well known that such a Lie algebra isomorphism is induced either by an isomorphism of vector spaces either between $W_{1}$ or $W_{2}$ or between $W_{1}^{*}=\operatorname{Hom}_{C}\left(W_{1}, C\right)$ and $W_{2}$. In the former case the spectra of $f_{1}$ and $f_{2}$ coincide (including the multiplicities). In the latter case the spectra of $-f_{1}$ and $f_{2}$ coincide (including the multiplicities). This implies that either $a_{1}=a_{2}$ or $a_{1}=n-a_{2}$. This contradicts our assumptions and proves the case of $d=2$. In the case of arbitrary $d \geq 2$, let us apply Lemma 3.5 to the image of $\mathfrak{k}^{s s}$ in $\mathfrak{s l}\left(W_{i}\right) \oplus \mathfrak{s l}\left(W_{j}\right)$ : we obtain that for every pair of distinct indices $i, j \leq d$ the projection map $\mathfrak{k}^{s s} \rightarrow \mathfrak{s l}\left(W_{i}\right) \oplus \mathfrak{s l}\left(W_{j}\right)$ is surjective. Now the case of arbitrary $d$ follows from Lemma on pp. 790-791 of [4].

Proof of Theorem 3.4. Let us split the reductive $Q$-Lie algebra $\mathfrak{k}_{C}$ Lie into a direct sum

$$
\mathfrak{k}_{C}=\mathfrak{k}^{s s} \oplus \mathfrak{c}
$$

of its center $\mathfrak{c} \subset E$ and the semisimple $Q$-Lie algebra

$$
\mathfrak{k}^{s s}=[\mathfrak{k}, \mathfrak{k}]=[\mathfrak{k}, \mathfrak{k}]
$$

Then $f=f_{0}+f_{\mathfrak{c}}$ with $f_{0} \in \mathfrak{k}_{C}^{s s}, f_{\mathfrak{c}} \in \mathfrak{c}_{C}$. By Remark 3.2, the $\mathfrak{k}_{C}$-module $V_{\sigma}$ is absolutely simple for all $\sigma \in \Sigma$. By Schur's Lemma there exists $c_{\sigma} \in C$ such that $f_{\mathfrak{c}}$ acts in $V_{\sigma}$ as multiplication by $c_{\sigma}$. It follows that $f_{0}$ acts in $V_{\sigma}$ as a diagonalizable operator with eigenvalues $\lambda-c_{\sigma}$ of positive multiplicity $n_{\sigma}$ and $\mu-c_{\sigma}$ of positive multiplicity $m_{\sigma}$. Let us denote by $f_{\sigma}$ the linear operator in $V_{\sigma}$ induced by $f_{0}$. It follows from Remark 3.2 that

$$
f_{\sigma} \in\left\{E \mathfrak{k}^{s s}\right\}_{\sigma} \subset \operatorname{End}_{C}\left(V_{\sigma}\right)
$$

and $\left\{E^{s s}\right\}_{\sigma}$ is an irreducible linear semisimple Lie $C$-(sub)algebra. It is also clear that $f_{\sigma}$ is a diagonalizable operator with (exactly two) eigenvalues $\lambda-c_{\sigma}$ of multiplicity $n_{\sigma}$ and $\mu-c_{\sigma}$ of multiplicity $m_{\sigma}$.

Taking $\sigma=\tau$ and applying Lemma 3.3 to $W=W_{\tau}, f=f_{\tau}$ and $\mathfrak{g}=\left\{E \mathfrak{k}^{s s}\right\}_{\sigma}$, we conclude that $\left\{E \mathfrak{k}^{s s}\right\}_{\tau}=\mathfrak{s l}\left(W_{\tau}\right)$. In other words, the image of $\mathfrak{k}_{C}^{s s} \rightarrow \operatorname{End}_{C}\left(V_{\tau}\right)$ coincides with $\mathfrak{s l}\left(V_{\tau}\right)$; in particular, the $C$-dimension of the image is $h^{2}-1$. By Remark 3.1, for all $\sigma \in C$ the image of $\mathfrak{k}_{C}^{s s} \rightarrow \operatorname{End}_{C}\left(V_{\sigma}\right)$ also has $C$-dimension $h^{2}-1$. The semisimplicity of $\mathfrak{k}_{C}^{s s}$ implies the semisimplity of the image and therefore this image must lie in $\mathfrak{s l}\left(V_{\sigma}\right)$; in particular, its $C$-dimension does not exceed $h^{2}-1$ (recall that $\left.h=\operatorname{dim}_{C}\left(V_{\sigma}\right)\right)$. It follows that the image of $\mathfrak{k}_{C}^{s s} \rightarrow \operatorname{End}_{C}\left(V_{\sigma}\right)$ coincides with $\mathfrak{s l}\left(V_{\sigma}\right)$ for all $\sigma \in \Sigma$.

Now let us choose a maximal subset $\Pi \subset \Sigma$ with respect to the following property:

$$
n_{\sigma} \neq m_{\kappa} \forall \sigma, \kappa \in \Pi \text {. }
$$

We claim that $\#(\Pi) \geq \#(\Sigma) / 2$. Indeed, if $\#(\Pi)<\#(\Sigma) / 2$ then the cardinality of the set

$$
N_{\Pi}=\left\{n_{\sigma} \mid \sigma \in \Pi\right\} \cup\left\{m_{\sigma} \mid \sigma \in \Pi\right\}
$$


does not exceed

$$
2 \cdot \#(\Pi)<2 \cdot \frac{\#(\Sigma)}{2}=\#(\Sigma) .
$$

This implies that $\#\left(N_{\Pi}\right)<\#(\Sigma)$ and therefore there exists $\kappa \in \Sigma$ such that $n_{\kappa}$ does not belong to $N_{\Pi}$; in particular, $\kappa$ does not belong to $\Pi$. It follows from the very definition of $N_{\Pi}$ that $m_{\kappa}$ also does not belong to $N_{\Pi}$. This implies that $\Pi$ is not maximal, because we could replace it by $\Pi \cup\{\kappa\}$. The obtained contradiction proves the desired inequality.

Since $\#(\Sigma)=[E: Q]$, we have $\#(\Pi) \geq[E: Q] / 2$. Now let us put

$$
W:=\oplus_{\sigma \in \Pi} V_{\sigma}
$$

and denote by $\mathfrak{k}_{\Pi}$ the image of $\mathfrak{k}_{C}^{s s}$ in

$$
\oplus_{\sigma \in \Pi} \operatorname{End}_{C}\left(V_{\sigma}\right) \subset \operatorname{End}_{C}\left(\oplus_{\sigma \in \Pi} V_{\sigma}\right)=\operatorname{End}_{C}(W) .
$$

Clearly, the linear operator $f_{\Pi}: W \rightarrow W$ induced by $f$ is a semisimple element of $\mathfrak{k}_{\Pi}$. Now the result follows from Lemma 3.6 applied to $W$, the semisimple $C$-Lie algebra $\mathfrak{k}_{\Pi} \subset \operatorname{End}_{C}(W)$ and $f_{\Pi}$.

The following statement will be used in Section 4 .

Lemma 3.7. Let $r \geq 2$ be a positive integer and let $\mathfrak{g}_{1}, \ldots, g_{r}$ be mutually nonisomorphic finite-dimensional simple $Q$-Lie algebras. Let $\mathfrak{g} \subset \oplus_{i=1}^{r} \mathfrak{g}_{i}$ be a semisimple Q-Lie subalgebra such that every projection map $\mathfrak{g} \rightarrow \mathfrak{g}_{i}$ is surjective. Then $\mathfrak{g}=\oplus_{i=1}^{r} \mathfrak{g}_{i}$

Proof. Let $i, j \leq r$ be two distinct positive integers. Applying Lemma 3.5 to the image of $\mathfrak{g}$ in $\mathfrak{g}_{i} \oplus \mathfrak{g}_{j}$ (with respect to the corresponding projection map), we conclude that the projection map $\mathfrak{g} \rightarrow g_{i} \oplus \mathfrak{g}_{j}$ is surjective. Now the result follows from Lemma on pp. $790-791$ of [4].

\section{Semisimple components of Hodge groups and their Lie algebras}

Proof of Theorem 1.1. Let us apply Theorem 3.4 to

$$
\begin{gathered}
Q=\mathbf{Q}, C=\mathbf{C}, V=\mathrm{H}_{1}(Z, \mathbf{Q}), h=d(Z, E), \\
\mathfrak{k}=\mathrm{hdg}, f=\mathfrak{f}_{H}^{0}, \lambda=-\frac{1}{2}, \mu=\frac{1}{2}, \\
n_{\sigma}=n_{\sigma}(Z, E), m_{\sigma}=d(Z, E)-n_{\sigma}(Z, E)=n_{\bar{\sigma}}(Z, E) .
\end{gathered}
$$

We conclude that

$$
\operatorname{dim}_{\mathbf{Q}}\left(\operatorname{hdg}^{s s}\right) \geq \frac{1}{2}[E: \mathbf{Q}]\left\{d(Z, E)^{2}-1\right\} .
$$

By Remark 1.2, this implies that

$$
\operatorname{hdg}^{s s}=\mathfrak{s u}\left(\mathrm{H}_{1}(Z, \mathbf{Q}), \phi\right)
$$

and we are done. 
4.1. Let us assume that $p$ is odd and $q=p^{r}$ and consider the abelian variety $Z=$ $\prod_{i=1}^{r} J^{\left(f, p^{i}\right)}$ and its first rational homology group $\mathrm{H}_{1}(Z, \mathbf{Q})=\oplus_{i=1}^{r} \mathrm{H}_{1}\left(J^{\left(f, p^{i}\right)}, \mathbf{Q}\right)$. Then every subspace $\mathrm{H}_{1}\left(J^{\left(f, p^{i}\right)}, \mathbf{Q}\right)$ is $\operatorname{Hdg}(Z)$-invariant and the image of $\operatorname{Hdg}(Z)$ in $\mathrm{GL}_{\mathbf{Q}}\left(\mathrm{H}_{1}\left(J^{\left(f, p^{i}\right)}, \mathbf{Q}\right)\right)$ coincides with $\operatorname{Hdg}\left(J^{\left(f, p^{i}\right)}\right)$. (This assertion follows easily from the minimality property of Hodge groups.) It follows that every $\mathrm{H}_{1}\left(J^{\left(f, p^{i}\right)}, \mathbf{Q}\right)$ is $\operatorname{hdg}_{Z}$-invariant and the image of $\operatorname{hdg}_{Z}$ in $\operatorname{End}_{\mathbf{Q}}\left(\mathrm{H}_{1}\left(J^{\left(f, p^{i}\right)}, \mathbf{Q}\right)\right)$ coincides with the Lie algebra of $\operatorname{Hdg}\left(J^{\left(f, p^{i}\right)}\right)$. This implies that the image of the semisimple Q-Lie algebra $\operatorname{hdg}_{Z}^{s s}$ in $\operatorname{End}_{\mathbf{Q}}\left(\mathrm{H}_{1}\left(J^{\left(f, p^{i}\right)}, \mathbf{Q}\right)\right)$ coincides with the semisimple part of the Lie algebra of $\operatorname{Hdg}\left(J^{\left(f, p^{i}\right)}\right)$.

Theorem 4.2. Suppose that $p$ is an odd prime, $n \geq 4$ is a positive integer such that $p$ does not divide $n(n-1)$. Suppose that $f(x) \in \mathbf{C}[x]$ is a degree $n$ polynomial without multiple roots. Suppose that there exists a subfield $K$ of $\mathbf{C}$ that contains all the coefficients of $f(x)$. Let us assume that $f(x)$ is irreducible over $K$ and the Galois group $\mathrm{Gal}(f)$ of $f(x)$ over $K$ is either $\mathbf{S}_{n}$ or $\mathbf{A}_{n}$. Assume additionally that either $n \geq 5$ or $n=4$ and $\operatorname{Gal}(f)=\mathbf{S}_{4}$. Let $r$ be a positive integer and $q=p^{r}$. Let us put $Z=\prod_{i=1}^{r} J^{\left(f, p^{i}\right)}$. If $n>q$ then

$$
\operatorname{hdg}_{Z} s s=\oplus_{i=1}^{r} \mathfrak{s u}\left(\mathrm{H}_{1}\left(J^{\left(f, p^{i}\right)}, \mathbf{Q}\right), \phi_{p^{i}}\right) .
$$

Proof. It follows from Theorem 0.1 that the semisimple part of the Lie algebra of $\operatorname{Hdg}\left(J^{\left(f, p^{i}\right)}\right)$ coincides with $\mathfrak{s u}\left(\mathrm{H}_{1}\left(J^{\left(f, p^{i}\right)}, \mathbf{Q}\right), \phi_{p^{i}}\right)$ for all $i \leq r$; notice that all $\mathfrak{s u}\left(\mathrm{H}_{1}\left(J^{\left(f, p^{i}\right)}, \mathbf{Q}\right), \phi_{p^{i}}\right)$ 's are mutually nonisomorphic simple Q-Lie algebras. Now the result follows from arguments of Subsect. 4.1 combined with Lemma 3.7.

We keep the notation and assumptions of Theorem 4.2. For every positive integer $i$ let us put

$\mathbf{Q}\left(\zeta_{p^{i}}\right)_{-}:=\left\{e \in \mathbf{Q}\left(\zeta_{p^{i}}\right) \mid \bar{e}=-e\right\} \subset \mathbf{Q}\left(\zeta_{p^{i}}\right)=\operatorname{End}^{0}\left(J^{\left(f, p^{i}\right)}\right) \subset \operatorname{End}_{\mathbf{Q}}\left(\mathrm{H}_{1}\left(J^{\left(f, p^{i}\right)}, \mathbf{Q}\right)\right)$.

Let us put

$$
\begin{gathered}
\operatorname{Tr}_{i}=\operatorname{Tr}_{\mathbf{Q}\left(\zeta_{p^{i+1}}\right) / \mathbf{Q}\left(\zeta_{p^{i}}\right): \mathbf{Q}\left(\zeta_{p^{i+1}}\right) \rightarrow \mathbf{Q}\left(\zeta_{p^{i}}\right)} \\
\mathrm{E}_{-}^{p, r}:=\left\{\left(e_{i}\right)_{i=1}^{r} \in \oplus_{i=1}^{r} \mathbf{Q}\left(\zeta_{p^{i}}\right)_{-} \mid \operatorname{Tr}_{i}\left(e_{i+1}\right)=e_{i} \forall i<r\right\} \subset \\
\oplus_{i=1}^{r} \mathbf{Q}\left(\zeta_{p^{i}}\right)_{-} \subset \oplus_{i=1}^{r} \operatorname{End}_{\mathbf{Q}}\left(\mathrm{H}_{1}\left(J^{\left(f, p^{i}\right)}, \mathbf{Q}\right)\right) \subset \operatorname{End}_{\mathbf{Q}}\left(\mathrm{H}_{1}(Z, \mathbf{Q})\right) .
\end{gathered}
$$

Recall [9] that (under our assumptions) the center of the $\mathbf{Q}$-Lie algebra hdg $_{Z}$ coincides with

$$
\mathrm{E}_{-}^{p, r} \subset \operatorname{End}_{\mathbf{Q}}\left(\mathrm{H}_{1}(Z, \mathbf{Q})\right) .
$$

Then the reductiveness of $\operatorname{hdg}_{Z}$ combined with Theorem 4.2 implies the following statement.

Theorem 4.3. Suppose that $p$ is an odd prime, $n \geq 4$ is a positive integer such that $p$ does not divide $n(n-1)$. Suppose that $f(x) \in \mathbf{C}[x]$ is a degree $n$ polynomial without multiple roots. Suppose that there exists a subfield $K$ of $\mathbf{C}$ that contains all the coefficients of $f(x)$. Let us assume that $f(x)$ is irreducible over $K$ and the Galois group $\operatorname{Gal}(f)$ of $f(x)$ over $K$ is either $\mathbf{S}_{n}$ or $\mathbf{A}_{n}$. Assume additionally that either 
$n \geq 5$ or $n=4$ and $\operatorname{Gal}(f)=\mathbf{S}_{4}$. Let $r$ be a positive integer and $q=p^{r}$. Let us put $Z=\prod_{i=1}^{r} J^{\left(f, p^{i}\right)}$. If $n>q$ then

$$
\operatorname{hdg}_{Z}=\mathrm{E}_{-}^{p, r} \oplus\left[\oplus_{i=1}^{r} \mathfrak{s u}\left(\mathrm{H}_{1}\left(J^{\left(f, p^{i}\right)}, \mathbf{Q}\right), \phi_{p^{i}}\right)\right] .
$$

Remark 4.4. We keep the assumptions of Theorem 4.3. Let us fix an isogeny $\alpha$ : $J\left(C_{f, p^{r}}\right) \rightarrow \prod_{i=1}^{r} J^{\left(f, p^{i}\right)}=Z$. Then $\alpha$ induces an isomorphism of Q-vector spaces $\alpha: \mathrm{H}_{1}\left(J\left(C_{f, p^{r}}\right), \mathbf{Q}\right) \cong \mathrm{H}_{1}(Z, \mathbf{Q})$. Clearly, the Hodge group of $\operatorname{Hdg}\left(J\left(C_{f, p^{r}}\right)\right)$ coincides with $\alpha^{-1} \operatorname{Hdg}(Z) \alpha$. This implies that the Q-Lie algebra of $\left.\operatorname{Hdg}\left(J\left(C_{f, p^{r}}\right)\right)\right)$ coincides with

$$
\alpha\left\{\mathrm{E}_{-}^{p, r} \oplus\left[\oplus_{i=1}^{r} \mathfrak{s u}\left(\mathrm{H}_{1}\left(J^{\left(f, p^{i}\right)}, \mathbf{Q}\right), \phi_{p^{i}}\right)\right]\right\} \alpha^{-1} .
$$

\section{References}

[1] P. Deligne, Hodge cycles on abelian varieties (notes by J.S. Milne). Lecture Notes in Math., vol. 900 (Springer-Verlag, 1982), pp. 9-100.

[2] B. Moonen, Yu. G. Zarhin, Weil classes on abelian varieties. J. reine angew. Math. 496 (1998), 83-92.

[3] D. Mumford, Abelian varieties, Second edition. Oxford University Press, London, 1974.

[4] K. Ribet, Galois action on division points of Abelian varieties with real multiplications. Amer. J. Math. 98, 751-804 (1976).

[5] K. Ribet, Hodge classes on certain abelian varieties. Amer. J. Math. 105 (1983), 523-538.

[6] J.-P. Serre, Sur les groupes de Galois attachés aux groupes p-divisibles. Proc. Conf. Local Fields (Driebergen, 1966), pp. 118-131, Springer, Berlin, 1967.

[7] J.-P. Serre, Représentations linéares des groupes finis, Troisiéme édition. Hermann, Paris, 1978.

[8] G. Shimura, Abelian varieties with complex multiplication and modular functions. Princeton University Press, Princeton, 1997.

[9] J. Xue, Yu.G. Zarhin, Centers of Hodge groups of superelliptic jacobians. arXiv:0907.1563 [math.AG]; to appear in Transformation Groups.

[10] Yu.G. Zarhin, Weights of simple Lie algebras in the cohomology of algebraic varieties. Izv. Akad. Nauk SSSR Ser. Mat. 48 (1984), 264-304; Math. USSR Izv. 24 (1985), 245 - 281.

[11] Yu.G. Zarhin, Linear irreducible Lie algebras and Hodge structures. Algebraic geometry (Chicago, IL, 1989), 281-297, Lecture Notes in Math. 1479, Springer, Berlin, 1991.

[12] Yu.G. Zarhin, Hyperelliptic jacobians without complex multiplication. Math. Res. Letters 7 (2000), 123-132.

[13] Yu.G. Zarhin, Very simple 2-adic representations and hyperelliptic jacobians. Moscow Math. J. 2 (2002), issue 2, 403-431.

[14] Yu.G. Zarhin, Cyclic covers, their Jacobians and endomorphisms. J. reine angew. Math. 544 (2002), 91-110.

[15] Yu.G. Zarhin, The endomorphism rings of Jacobians of cyclic covers of the projective line. Math. Proc. Cambridge Philos. Soc. 136 (2004), 257-267.

[16] Yu.G. Zarhin, Endomorphism algebras of superelliptic Jacobians. In: F. Bogomolov, Yu. Tschinkel (eds.) Geometric methods in Algebra and Number Theory, Progress in Math. 235 339-362, Birkhäuser, Boston Basel Berlin, 2005.

[17] Yu.G. Zarhin, Superelliptic jacobians. In: "Diophantine Geometry" Proceedings (U. Zannier, ed.), Edizioni Della Normali, Pisa 2007, pp. 363-390.

[18] Yu.G. Zarhin, Endomorphisms of superelliptic jacobians. Math. Z., 261 (2009), 691-707, 709.

Department of Mathematics, Pennsylvania State University, University Park, PA 16802, USA

E-mail address: xue_j@math.psu.edu

Department of Mathematics, Pennsylvania State University, University Park, PA 16802, USA

E-mail address: zarhin@math.psu.edu 